\title{
Extreme Tracheal Compression due to Substernal Goiter: Surgical and Anesthetic Management
}

\author{
Benzon Dy, Kevin Wise, David Farley, Brian McGlinch
}

\begin{abstract}
Substernal goiters can have a range of presentations and pathology from a complete lack of symptoms to minimal biochemical abnormalities to potentially lethal physical derangements due to tracheal compression. We review a case of a substernal goiter with severe tracheal compression and airway compromise and describe the presentation, diagnosis, operative treatment, perianesthetic management and outcome of this patient. Substernal goiters can have a myriad of symptoms and etiologies. Once the diagnosis is secured, a multidisciplinary approach is key to successful management in the perioperative period.
\end{abstract}

Keywords: Goiter, Thyroid, Tracheal compression.

How to cite this article: Dy B, Wise K, Farley D, McGlinch B. Extreme Tracheal Compression due to Substernal Goiter: Surgical and Anesthetic Management. World J Endocr Surg 2012;4(2):71-73.

\section{Source of support: Nil}

Conflict of interest: None declared

\section{INTRODUCTION}

Substernal goiters occur when a thyroid mass descends below the thoracic inlet. Up to $45 \%$ of patients with a goiter may have a substernal component. ${ }^{1}$ Enlargement of the thyroid gland with a substernal component may be asymptomatic or have a myriad of presentations due to compression of surrounding structures. Patients may present with dyspnea, wheezing, severe respiratory compromise and dysphagia. ${ }^{2,3}$ Occlusion of the SVC when raising one's arms may result in venous congestion manifesting with symptoms of flushing, stridor and elevation of internal jugular pressure known as the Pemberton sign. ${ }^{4}$ Dysphagia and dysphonia may result due to compression of the recurrent laryngeal nerve. Substernal goiters can also present with features of hyperthyroidism, especially if long-standing. Here, we describe a case of a substernal goiter with resultant severe tracheal compression and airway compromise.

\section{CASE REPORT}

A 57-year-old man presented to an emergency department with stridor and wheezing. He had a 6-month history of respiratory difficulty and was treated by his primary care physician with bronchodilators with no improvement. In the emergency department, a chest X-ray was performed showing right-sided deviation of his trachea. A computed tomography (CT) scan with scout imaging (Fig. 1) demonstrated severe compression of his trachea to $2 \mathrm{~mm}$ secondary to substernal extension of an enlarged thyroid gland (Fig. 2). He was emergently transferred to our institution.

Preoperative management included an echocardiogram and beta-blockade secondary to his history of coronary artery disease. Due to the patient's unstable airway, an urgent multidisciplinary evaluation was performed by pulmonology, anesthesiology and general surgery staff. The patient's airway was evaluated and secured with direct bronchoscopy under conscious sedation in the operating room while maintaining spontaneous ventilation. The trachea was intubated safely with a $7 \mathrm{~mm}$ endotracheal tube and general anesthesia was administered.

A standard cervical collar incision was made and the thyroid was exposed. Gross examination of the thyroid revealed a large and cystic gland extending from high in the neck to the thoracic inlet. The intrathoracic component of the thyroid was mobilized into the neck and a left thyroid lobectomy and isthmusectomy were performed (Fig. 3). Blood loss was minimal. Direct laryngoscopy ruled out tracheomalacia postoperatively. Frozen section histology showed a benign cystic thyroid with no evidence of malignancy. Permanent histopathological sections confirmed the diagnosis of benign multinodular goiter.

The patient did well postoperatively with no signs of tracheomalacia and had complete resolution of his symptoms. He was dismissed on postoperative day number two.

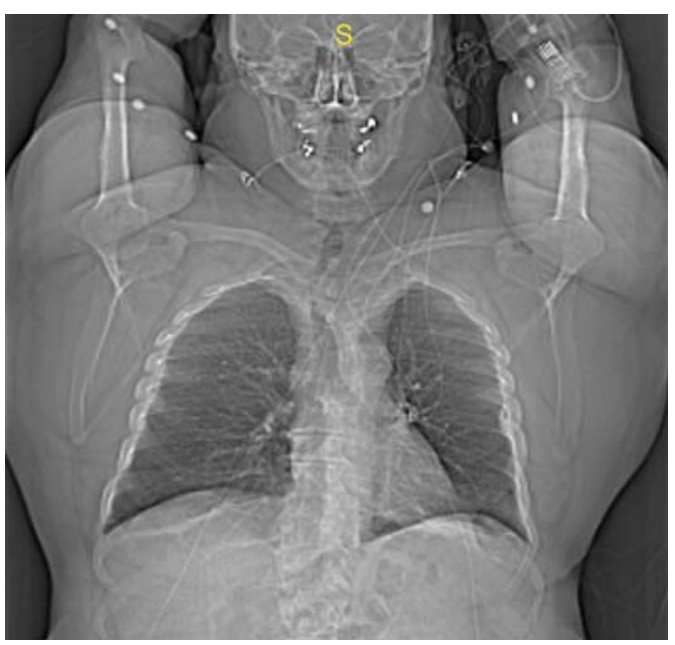

Fig. 1: Anterior poster scout image of the neck demonstrating tracheal compression and narrowing of the lumen 


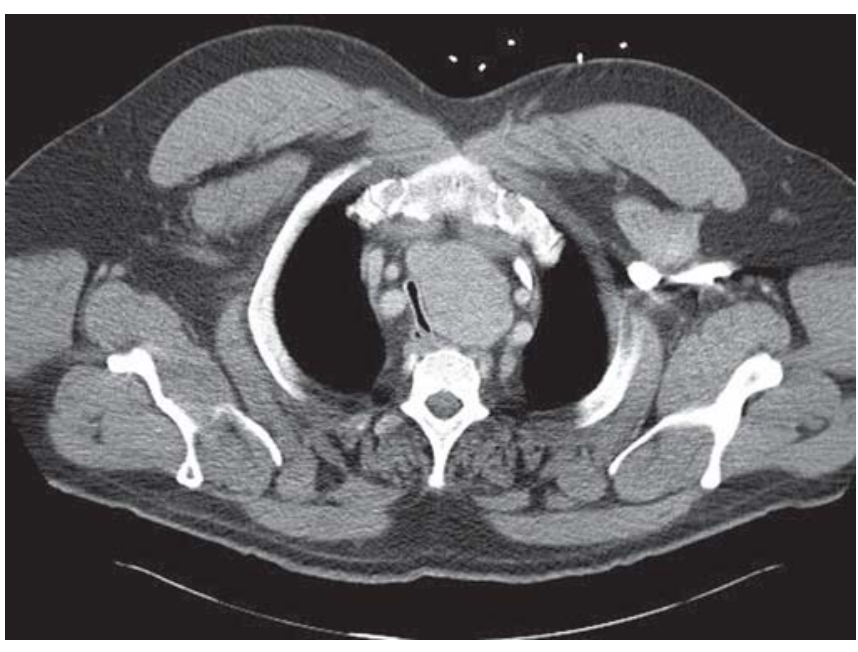

Fig. 2: Axial computed tomography (CT) scan demonstrating shift and compression of the trachea to $2 \mathrm{~mm}$ due to the substernal component of the thyroid

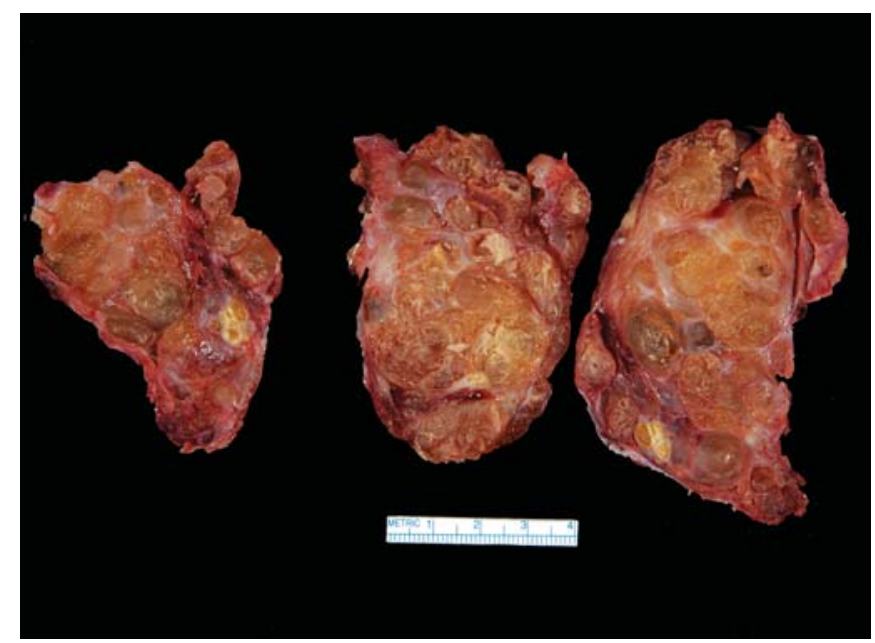

Fig. 3: Resected left thyroid lobe and isthmus

\section{DISCUSSION}

Tracheal compression with subsequent inability to ventilate is a feared complication of substernal goiters. Although a tracheostomy or emergent cricothyrotomy may be considered, the location of this goiter would make tracheostomy technically challenging and potentially impossible to perform in time to prevent hypoxia-induced complications. The increased size of the thyroid parenchyma would hinder dissection to the trachea and increase the distance to the tracheostomy tube would need to travel to safely intubate the trachea. The tracheal lumen would be difficult to cannulate due to external pressure and its decreased cross-sectional area.

The anesthetic management for patients with substernal goiters requires a careful review of patient symptoms and imaging studies prior to induction of anesthesia. Asymptomatic patients without dyspnea at rest or dysphagia are unlikely to have significant goiter-related tracheal compression. When the goiter compresses a normal tracheal diameter by 50 to $70 \%$, dyspnea on exertion develops. Goiter compression of a normal trachea beyond $70 \%$ of its usual diameter results in dyspnea at rest and represents the highest risk patient for airway management complications. ${ }^{5}$ Imaging studies, including chest X-rays and cervical CT scans should be reviewed as part of the surgical and anesthetic preoperative assessment, particularly for those patients with dyspnea. The chest X-ray may demonstrate the presence of tracheal distortion related to the goiter. CT scans of the neck and chest will demonstrate the length and degree of tracheal compression but also the presence of fat (or other compressible tissue) at the site of the most significant stenosis. This information is critical in determining if the airway can expand to accommodate an endotracheal tube. Clinical situations where the goiter (or other noncompressible tissue) surrounds the intrathoracic trachea at the area of stenosis (rendering the passage of an endotracheal tube impossible) warrants the use of considerably more invasive interventions, such as the availability of extracorporeal circulation to ensure oxygenation during the operation. Fortunately, interventions of this magnitude are rarely necessary during operative management of thyroid disease.

Tracheal intubation in the presence of intrathoracic tracheal compression is facilitated by fiberoptic bronchoscopy performed while the patient is spontaneously breathing. Direct visualization of the distal airway using fiberoptic bronchoscopy affords precise placement of the endotracheal tube distal to the stenotic tracheal segment. When a patient with intrathoracic tracheal compression is paralyzed prior to placement of an endotracheal tube, positive pressure ventilation potentially compromises the airway at the stenosis site. The tracheal walls are drawn together during positive pressure ventilation causing airway collapse and the patient cannot be ventilated.

The importance of maintaining spontaneous ventilation during bronchoscopy-facilitated placement of an endotracheal tube cannot be understated. Additionally, tracheal extubation following intrathoracic goiter resection warrants some consideration. Although there is a risk of postoperative tracheomalacia after resection of intrathoracic goiters, a review of multiple studies show the incidence ranges from $0 \%$ in seven series to $10.3 \%$ in one series. ${ }^{6}$ The rate of tracheostomy ranges from $0 \%$ in three series to $8.6 \%$ in one series. ${ }^{6}$ Patients typically breathe easier following goiter resection as the tracheal compression is alleviated and tracheal caliber returns to normal. A risk for laryngeal nerve injury following goiter resection also exists. Recurrent laryngeal nerve monitoring technology is readily available and often utilized by surgeons performing thyroid 
operations. The use of this technology may help to avoid recurrent laryngeal nerve injury during complicated neck operations. However, even with the use of nerve monitoring technology, tracheal extubation should be performed in the operating room to afford the safest patient environment, if vocal cord dysfunction is present.

Once the airway is secured, there are multiple options for surgical management of the thyroid. The gland is accessed through a cervical approach and can be delivered through the thoracic inlet into the neck in $98 \%$ of cases. ${ }^{7}$ This is typically achieved with gentle dissection of the inferior pole of the thyroid away from mediastinal tissues and subsequent cephalad elevation of the inferior pole of the thyroid into the neck. In instances with extensive intrathoracic involvement of the thyroid, an extension of the standard cervical incision inferiorly to the sternal notch is sometimes required to gain necessary access. Reports in the literature have described successful resection of goiters with extension below the aortic arch and to the level of the carina with this approach. ${ }^{8}$ When this is not possible, a mini-sternotomy or formal sternotomy may be performed. Careful attention should be directed toward preservation of the recurrent laryngeal nerve and parathyroid glands since substernal thyroidectomy is associated with a higher complication rate. ${ }^{9}$ Factors including tumor pathology, consistency of the gland and nodal involvement must also be assessed at the time of resection. The same oncologic principles of standard thyroid surgery apply to substernal goiters. However, the risk of malignancy is no higher than in other goiters. ${ }^{6}$

\section{CONCLUSION}

A multidisciplinary approach is essential for successful management of substernal goiters with severe tracheal compression. Imaging studies, particularly CT scans of the head, neck and chest are indicated for patients with evidence of dyspnea on exertion and necessary for patients with dyspnea at rest as a result of large substernal goiters. Early communication with anesthesia providers facilitates the safe airway management of these challenging patients.

\section{REFERENCES}

1. Hegedüs L, Bonnema SJ. Approach to management of the patient with primary or secondary intrathoracic goiter. Journal of Clinical Endocrinology and Metabolism 2010 Dec 1;95(12): 5155-62.

2. Newman E, Shaha AR. Substernal goiter. Journal of Surgical Oncology 1995;60(3):207-12.

3. Mellière D, Saada F, Etienne G, Becquemin J, Bonnet F. Goiter with severe respiratory compromise: Evaluation and treatment surgery 1988;103(3):367-73.

4. Pemberton H. Sign of submerged goitre. Lancet 1946; 248(6423):509.

5. Netterville JL, Coleman SC, Smith JC, Smith MM, Day TA, Burkey BB. Management of substernal goiter. The Laryngoscope 1998;108(11):1611-17.

6. White M, Doherty G, Gauger P. Evidence-based surgical management of substernal goiter. World Journal of Surgery 2008;32(7):1285-300.

7. Agha A, Glockzin G, Ghali N, Iesalnieks I, Schlitt H. Surgical treatment of substernal goiter: An analysis of 59 patients. Surgery Today 2008;38(6):505-11.

8. Rugiu MG, Piemonte M. Surgical approach to retrosternal goitre: Do we still need sternotomy? Acta Otorhinolaryngologica Italica 2009;29:331-38.

9. Pieracci FM, Fahey TJ. Substernal thyroidectomy is associated with increased morbidity and mortality as compared with conventional cervical thyroidectomy. Journal of the American College of Surgeons 2007;205(1):1-7.

\section{ABOUT THE AUTHORS}

\section{Benzon Dy (Corresponding Author)}

Resident, Department of General Surgery, Mayo Clinic, Minnesota USA, e-mail: dy.benzon@mayo.edu

\section{Kevin Wise}

Resident, Department of General Surgery, Mayo Clinic, Minnesota USA

\section{David Farley}

Professor, Department of General Surgery, Mayo Clinic, Minnesota USA

\section{Brian McGlinch}

Professor, Department of Anesthesiology, Mayo Clinic, Minnesota, USA 